\title{
Étude expérimentale de la variabilité de la réponse à la sélection chez Drosophila melanogaster
}

\author{
C. LÓPEZ-FANJUL et María Luisa DOMíNGUEZ \\ Departamento de Genética, \\ Facultad de Biología, Universidad Complutense de Madrid, Ciudad Universitaria, \\ Madrid-3, Espagne
}

\begin{abstract}
Résumé
La réponse à court terme à la sélection pour la diminution du nombre de soies sternopleurales et sa variabilité ont été étudiées dans deux populations de Drosophila melanogaster (Dahomey et Kaduna) et pour deux taux de sélection différents $(10$ p. 100 et 35 p. 100), avec, dans chacun des quatre cas possibles, vingt lignées sélectionnées pendant 4-5 générations. Les valeurs observées du coefficient de variation de la réponse, ainsi que celles des coefficients de variation de la variance phénotypique cumulée ou non cumulée, sont raisonnablement en accord avec les prédictions correspondantes selon un modèle où la dérive génétique est le seul agent modificateur des fréquences génétiques des loci additifs et indépendants. En ce qui concerne le coefficient de variation de la variance phénotypique, la qualité de ces prédictions est détériorée quand l'effectif génétique des lignées sélectionnées est très bas. Donc, nos résultats sont en accord avec l'hypothèse selon laquelle la valeur de ces coefficients de variation dans des lignées sélectionnées à court terme peut être approximativement prédit suivant un modèle dans lequel il n'y a pas de sélection.
\end{abstract}

\section{Introduction}

L'étude expérimentale de la réponse à la sélection artificielle dans un ensemble de lignées extraites de la même population, et avec le même nombre d'individus mesurés et sélectionnés pour un même caractère dans chacune d'elles, a démontré l'existence d'une variation dans la réponse observée augmentant à mesure que le processus de sélection avance (Clayton et al., 1957 et López-FANJUl \& Hill, 1973, pour le nombre des soies abdominales et sternopleurales, respectivement, chez Drosophila melanogaster; FALCONER, 1973, pour le poids de la souris à six semaines ; TORO, 1978, pour la ponte de femelles vierges chez Tribolium castaneum).

Cette variabilité de la réponse peut être attribuée en partie à la dérive génétique, et aussi à l'action d'effets de milieu spécifiques propres à une lignée et à une génération déterminées. Hill $(1972 \mathrm{a}, \mathrm{b}, 1974,1977)$ a élaboré un modèle qui permet de prédire la variation de la réponse à la sélection pour un caractère régi par des gènes agissant de façon additive dont les variances génétique et phénotypique ne 
changent pas pendant la durée du processus de sélection, dans des lignées sélectionnées dont l'effectif génétique $(N)$ est suffisamment élevé. Le modèle peut être pratiquement appliqué à l'étude de la sélection à court terme $(N / 2$ générations) tant que la réponse se maintient linéaire. Quelques aspects de ce modèle ont été vérifiés expérimentalement par YuksEL (1974) lequel, utilisant des données de travaux antérieurs, trouva que l'accord entre l'observation et la prédiction dépend du caractère analysé ; cette étude était basée sur des expériences qui comptaient un petit nombre de lignées (six ou moins), où le taux de sélection était, en général, de l'ordre de 20 p. 100.

Dans ce travail, nous étudions la réponse à la sélection et spécialement sa variabilité, pour diminuer le nombre de soies sternopleurales, dans deux populations de $D$. melanogaster et avec deux taux de sélection différents, avec vingt lignées sélectionnées dans chacune des quatre situations possibles.

La prédiction théorique de la variabilité de la réponse à la sélection présente un intérêt réel puisque la validité d'un plan d'amélioration génétique dépend, non seulement de l'importance de la réponse espérée, mais aussi du biais impliqué dans la prédiction de cette réponse. La prise en considération de ce dernier facteur est importante, au moment de prendre la décision de mettre en pratique un plan de sélection déterminé.

\section{Matériel et méthodes}

Les populations de $D$. melanogaster utilisées ont été la Kaduna $(K)$ et la Dahomey $(D)$, récoltées en 1948 et 1973, respectivement, et maintenues depuis en conditions standard de laboratoire. Le caractère étudié est le nombre de soies sternopleurales mesuré à une température de $25^{\circ} \mathrm{C} \pm 0,5^{\circ} \mathrm{C}$ et une humidité relative de 70 p. 100 dans un milieu standard. Le déterminisme génétique additif de ce caractère a été démontré à plusieurs reprises (ROBERTSON, 1967, 1968).

Dans chaque population on considère deux groupes chacun de vingt lignées sélectionnées, qui correspondent à deux taux de sélection massale différents : 10 p. 100 (on a sélectionné dans chaque sexe les deux individus ayant le nombre le plus bas de soies parmi vingt individus mesurés, $2 / 20$; lignées $D_{2}$ et $K_{2}$ ) et 35 p. 100 $\left(7 / 20\right.$; lignées $D_{7}$ et $\left.K_{7}\right)$. Le nombre de générations de sélection a été, respectivement, de quatre et cinq pour les lignées avec un taux de sélection $2 / 20$ et $7 / 20$. Dans chaque population on a pris deux échantillons d'œufs au hasard, et à partir de chacun d'eux, on a obtenu 400 mâles et 400 femelles vierges ; ces individus ont été répartis au hasard dans vingt groupes de 20 mâles et 20 femelles chacun, qui ont constitué la génération 0 des vingt lignées sélectionnées dans chacune des quatre situations étudiées $\left(\mathrm{D}_{7}, \mathrm{~K}_{7}, \mathrm{D}_{2}\right.$ et $\left.\mathrm{K}_{2}\right)$.

On a calculé l'héritabilité des populations Kaduna et Dahomey par le coefficient de régression linéaire de la valeur moyenne des fils sur la valeur moyenne des parents, suivant la méthode de HILl (1970) afin d'améliorer la précision statistique de l'estimation. Les héritabilités réalisées dans chaque lignée ont été calculées par le coefficient de régression linéaire de la réponse cumulée sur la différentielle de sélection cumulée. Une héritabilité réalisée globale a été calculée pour chaque groupe 
de lignées par le coefficient de régression linéaire de la réponse cumulée moyenne sur la valeur moyenne de la différentielle de sélection cumulée, et ses écarts-types ont été corrigés pour les erreurs d'estimation dues à la fois à l'échantillonnage des lignées et à la dérive génétique (HILl, 1972 b).

La prédiction de la variabilité de la réponse à la sélection a été faite d'après le modèle de HiLl (1974), où l'on considère un ensemble de lignées, extraites, toutes, de la même population, dans lesquelles on sélectionne $N / 2$ individus de chaque sexe à partir de $\mathbf{M} / 2$ candidats à la sélection mesurés, avec une intensité de sélection $i$ correspondant à un taux de sélection $N / M$, pendant $t$ générations. Pour un caractère d'héritabilité $h^{2}$ et de variance phénotypique $s^{2}$, la variance espérée des moyennes des lignées dans la génération $t, V\left(\bar{X}_{t}\right)$, est donné par l'expression :

$$
V\left(\bar{X}_{t}\right)=t h^{2} s^{2}\left[1-h^{2}\left(1-k_{p}\right)\right] / N+s^{2} / M
$$

$k_{p}$ étant une valeur dépendant du taux de sélection, qui représente le fait que $V\left(\bar{X}_{t}\right)$ ne dépend pas des différentielles de sélection appliquées. Le premier terme de la formule représente l'effet de la dérive génétique, qui est cumulé tout au long du processus sélectif, et le second provient de ce que la valeur génétique moyenne des individus sélectionnés à chaque génération est estimée à travers un échantillon de $M$ de ses fils, facteur qui n'est pas cumulable. Le coefficient de variation espéré de la réponse $C V\left(\bar{X}_{t}\right)$ sera donné par:

$$
C V\left(\bar{X}_{t}\right)=\sqrt{V\left(\bar{X}_{t}\right)} / t i h^{2} s
$$

S'il n'y a pas de sélection $V\left(\bar{X}_{t}\right)$ est donné par (WrIGHT, 1952) :

$$
V\left(X_{t}\right)=s^{2}\left[1+h^{2} F_{t}(2 M-1)\right] / M
$$

$F_{t}$ étant le coefficient de consanguinité qui correspond à la génération $t$, qui est donné par :

$$
F_{t}=1-(1-1 / 2 N)^{t}
$$

\section{Résultats}

\section{1. - Evolution des moyennes}

Les paramètres des populations sont portés dans le tableau 1. Les estimations de la moyenne, variance phénotypique et héritabilité de la population Dahomey sont supérieures à celles de la population Kaduna.

L'évolution de la moyenne par génération pour l'ensemble des vingt lignées correspondant à chacun des quatre cas étudiés est présentée dans la figure 1-1. La réponse moyenne reste pratiquement linéaire pendant les cinq générations de sélection effectuées dans les groupes de lignées $D_{7}$ et $K_{7}$, et pendant les deux premières générations dans les groupes $D_{2}$ et $K_{2}$. Pour un même taux de sélection les réponses moyennes observées dans les deux populations, qui ont été estimées par le coefficient de régression linéaire de la valeur moyenne pour le caractère sur le numéro de génération, ne sont pas significativement différentes $\left(D_{7}=0,56 \pm 0,07\right.$ vs. $K_{7}=0,61 \pm$ 

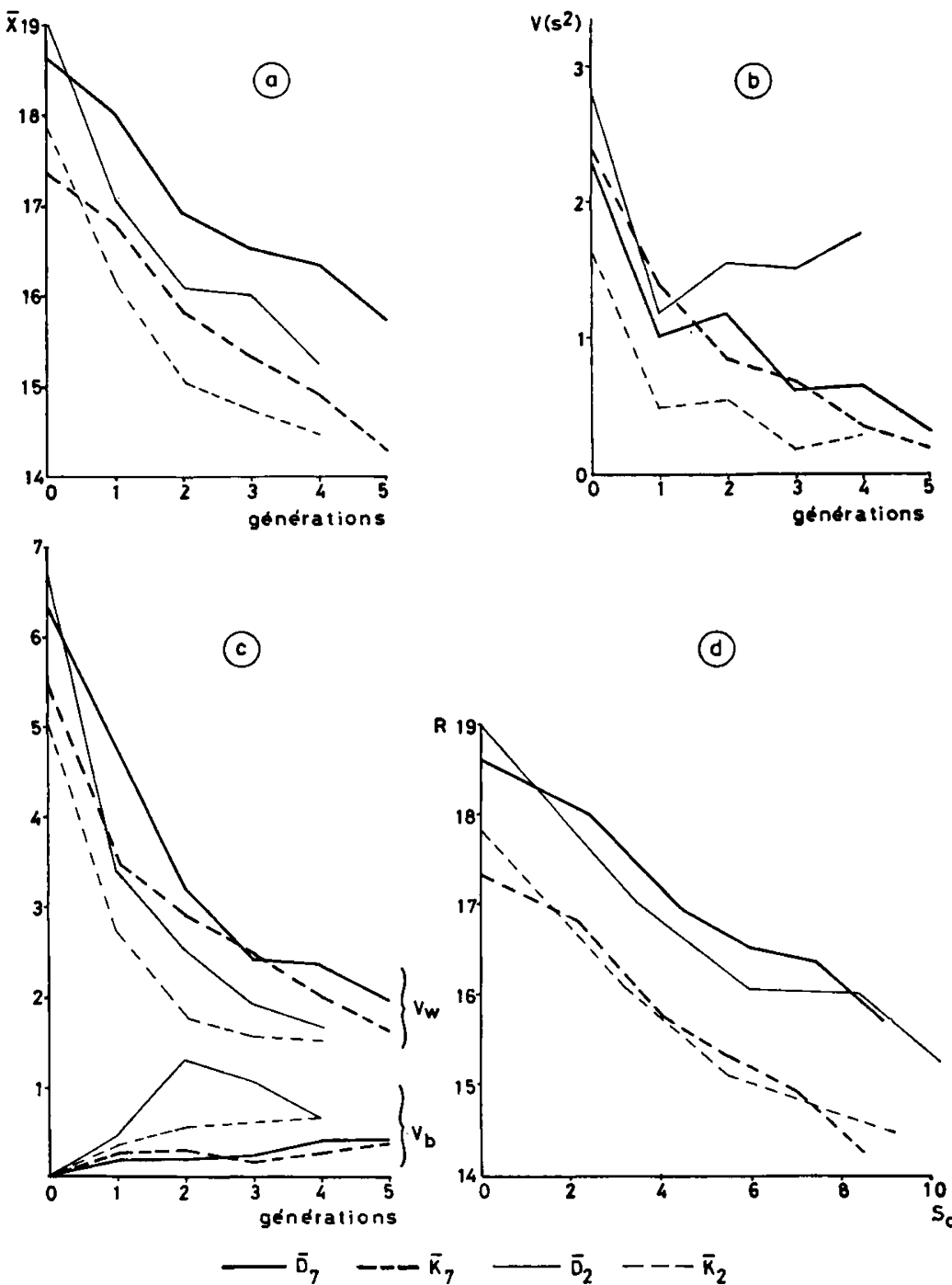

Fig. 1

a) Évolution des moyennes $(\overline{\mathrm{X}})$ des différents groupes de lignées.

Evolution of mean bristle number $(\overline{\mathrm{X}})$ for each group of lines.

b) Evolution des composantes intra lignée $\left(\mathrm{V}_{\mathrm{w}}\right)$ et inter lignées $\left(\mathrm{V}_{\mathrm{b}}\right)$ de la variance phénotypique, par groupe de lignées.

Evolution of the within- $\left(\mathrm{V}_{\mathrm{w}}\right)$ and between-line $\left(\mathrm{V}_{\mathrm{b}}\right)$ components of the phenotypic variance for each group of lines.

c) Évolution de la variance phénotypique $\left[\mathrm{V}\left(\mathrm{s}^{2}\right)\right]$ des différents groupes de lignées.

Evolution of the variance of the phenotypic variance $\left[\mathrm{V}\left(\mathrm{s}^{2}\right)\right]$ for each group of lines.

d) Réponse à la sélection $(\mathrm{R})$ en fonction de la différentielle de sélection cumulée $\left(\mathrm{S}_{\mathrm{c}}\right)$, par groupe de lignées.

Response to selection (R) plotted against cumulative selection differential $(\mathrm{Sc})$ for each group of lines. 
0,$04 ; D_{2}=0,86 \pm 0,19$ vs. $K_{2}=0,81 \pm 0,18$ ). Dans chaque population les lignées où le taux de sélection a été plus bas ont présenté une réponse plus élevée, en accord avec la prédiction de RoBERTSON (1970).

\section{TABleau 1}

Moyenne $(\bar{X})$, variance phénotypique $\left(s^{2}\right)$ et héritabilité $\left(h^{2}\right)$ des populations. Mean $(\bar{X})$, phenotypic variance $\left(s^{2}\right)$ and heritability $\left(h^{2}\right)$ of the populations.

\begin{tabular}{c|c|c|c}
\hline Population & $\bar{X} \pm s_{\bar{X}}$ & $s^{2}$ & $h^{2} \pm s_{h^{2}}$ \\
\hline Kaduna ..................... & $17,6 \pm 0,1$ & 5,2 & $0,39 \pm 0,05$ \\
Dahomey ....... & $18,8 \pm 0,1$ & 6,4 & $0,61 \pm 0,04$ \\
\hline
\end{tabular}

\section{2. - Evolution des variances}

La variance phénotypique par génération des lignées sélectionnées se réduit rapidement, pour atteindre à la fin de l'expérience une valeur de l'ordre de 30 p. 100 de sa valeur initiale. La figure $1 \mathrm{~b}$ présente les composantes entre lignées $\left(V_{b}\right)$ et intra lignée $\left(V_{w}\right)$ de la variance phénotypique où l'augmentation progressive de la première et la diminution drastique de la seconde apparaissent clairement. La réduction de la variance phénotypique a été plus importante dans les lignées où le nombre d'individus sélectionnés était plus bas, et pour cette raison elles devraient avoir un effectif génétique plus réduit. De toute façon, on a trouvé que cette réduction est considérablement supérieure à celle que l'on attendait sous les hypothèses du modèle infinitésimal, où l'on peut prévoir des diminutions dans la variance phénotypique de l'ordre de 8 p. 100 et 16 p. 100 pour des lignées avec un taux de sélection de 7/20 et $2 / 20$, respectivement (BULMER, 1971).

L'évolution de la variance phénotypique des lignées par génération est présentée dans la figure $1 \mathrm{c}$, où l'on observe une diminution très marquée de celle-là atteignant des valeurs finales de l'ordre de 10 à 15 p. 100 des valeurs initiales, exception faite des lignées $\mathrm{D}_{2}$ dans lesquelles cette diminution est de l'ordre de 60 p. 100 . Le coefficient de variation de la variance phénotypique est porté dans le tableau 2 par groupe de lignées et génération. S'il n’y a pas sélection, et en supposant que la variance due au milieu reste inchangée, la valeur espérée de ce coefficient dans la génération $t$, pour un caractère régi par des loci additifs et indépendants, est de $\sqrt{4\left[1-(1 / 4)^{t}\right]} \overline{l 3 N}$, qui tend rapidement vers $\sqrt{4 / 3 N}$ (HILL, 1977). Dans notre cas ces valeurs asymptotiques sont 32 p. 100 et 58 p. 100 pour des effectifs génétiques de 14 et 4, respectivement, et l'accord entre la prédiction et l'observation est acceptable dans le cas des lignées sélectionnées avec un taux de $7 / 20$, mais ce n'est pas le cas en ce qui concerne le taux $2 / 20$. 
TABleaU 2

Coefficient de variation (p. 100) de la variance phénotypique par groupe de lignées et génération.

Coefficient of variation (p. 100) of the phenotypic variance per group of lines and generation.

\begin{tabular}{|c|c|c|c|c|}
\hline \multirow{2}{*}{$\begin{array}{l}\text { Génération } \\
\text { Generation }\end{array}$} & \multicolumn{4}{|c|}{$\begin{array}{c}\text { Groupe de lignées } \\
\text { Group of lines }\end{array}$} \\
\hline & $\mathrm{D}_{7}$ & $\mathrm{~K}_{7}$ & $\mathrm{D}_{2}$ & $\mathbf{K}_{2}$ \\
\hline$\ldots \ldots \ldots \ldots \ldots \ldots$ & 24 & 29 & 25 & 26 \\
\hline$\ldots \ldots \ldots \ldots \ldots \ldots$ & 21 & 33 & 32 & 25 \\
\hline$\ldots \ldots \ldots \ldots \ldots$ & 34 & 31 & 49 & 41 \\
\hline$\ldots \ldots \ldots \ldots \ldots$ & 32 & 33 & 64 & 25 \\
\hline$\ldots \ldots \ldots \ldots \ldots \ldots$ & 34 & 29 & 80 & 34 \\
\hline $5 \ldots \ldots \ldots \ldots \ldots \ldots$ & 27 & 26 & - & 一 \\
\hline
\end{tabular}

Le coefficient de variation observé de la variance phénotypique cumulée jusqu'à la génération $t(t=1,2, \ldots, 5)$ est présenté dans le tableau 3 , pour chacun des quatre types de lignées. Selon les hypothèses que nous venons de donner, la valeur de ce coefficient doit rapidement atteindre le maximum pour diminuer ensuite (HILL, 1977). Nos données mettent en relief une réduction graduelle, dès le début. HrLL (1977) indique que l'on doit atteindre une valeur asymptotique de l'ordre de $\sqrt{1 / 3 N}$, ce qui correspond à 15 p. 100 et 20 p. 100 pour des effectifs génétiques de 14 et 4 , respectivement; encore une fois, on voit que nos données ont une précision acceptable pour des lignées sélectionnées avec le taux 7/20, mais ne l'ont pas avec le $\operatorname{taux} 2 / 20$.

\section{TABleaU 3}

Coefficient de variation (p. 100) de la variance phénotypique cumulée jusqu'à la génération $t$ par groupe de lignées.

Coefficient of variation ( $p .100$ ) of the cumulative phenotypic variance to generation $t$ for each group of lines.

\begin{tabular}{|c|c|c|c|c|}
\hline \multirow{2}{*}{$t$} & \multicolumn{4}{|c|}{$\begin{array}{l}\text { Groupe de lignées } \\
\text { Group of lines }\end{array}$} \\
\hline & $\mathrm{D}_{7}$ & $\mathbf{K}_{7}$ & $\mathrm{D}_{2}$ & $\mathrm{~K}_{2}$ \\
\hline$\ldots \ldots \ldots \ldots$ & 19 & 18 & 18 & 18 \\
\hline$\ldots \ldots \ldots \ldots \ldots \ldots$ & 14 & 16 & 16 & 16 \\
\hline$\ldots \ldots \ldots \ldots \ldots \ldots$ & 12 & 17 & 14 & 15 \\
\hline $4 \ldots \ldots \ldots \ldots \ldots$ & 11 & 16 & 13 & 14 \\
\hline $5 \ldots \ldots \ldots \ldots \ldots \ldots$ & 10 & 15 & - & - \\
\hline
\end{tabular}




\section{3. - Héritabilités réalisées}

Dans la figure 1-4, on présente les valeurs de la moyenne et de la différentielle de sélection cumulée par génération, dans chacun des quatre groupes de lignées; l'accord linéal a une précision très acceptable pendant la période considérée.

La valeur moyenne des vingt héritabilités réalisées $(\bar{b})$ estimées dans chaque groupe de lignées ainsi que celle estimée par le coefficient de régression linéaire de la réponse cumulée moyenne sur la valeur moyenne de la différentielle de sélection cumulée $\left(b_{c}\right)$ pour chaque groupe de lignées, sont présentées dans le tableau 4. Les deux estimations ne sont pas significativement différentes pour un groupe de lignées donné. Dans le tableau 4 figurent aussi trois valeurs de l'écart-type des estimations de l'héritabilité réalisée considérées : le premier $\left(s_{b}\right)$ est empirique et correspond à celui des vingt héritabilités réalisées estimées dans chaque groupe de lignées, le second $\left(s_{b c}\right)$ est celui du coefficient de régression $b_{c}$ et le troisième $\left(s_{b c}^{\prime}\right)$ est celui de l'héritabilité réalisée, estimé suivant la méthode proposée par HiLl (1972 b). Dans tous les cas, cela donne $s_{\bar{b}}<s_{b c}<s_{b c}^{\prime}$.

\section{TABLeaU 4}

Héritabilités réalisées moyennes $(\bar{b})$ et globales $\left(b_{c}\right)$ et ses écarts-types $\left(s_{\bar{b}}, s_{b c}, s_{b c}^{\prime}\right)$ par groupe de lignées.

Average $(\bar{b})$ and pooled $\left(b_{c}\right)$ realised heritabilities and their standard errors $\left(s_{\bar{b}}, s_{b c}, s_{b c}\right)$ for each group of lines.

\begin{tabular}{|c|c|c|c|c|c|}
\hline $\begin{array}{l}\text { Groupe de lignées } \\
\text { Group of lines }\end{array}$ & $\bar{b}$ & $b_{c}$ & $s_{\bar{b}}$ & $s_{b c}$ & $s^{\prime} b c$ \\
\hline 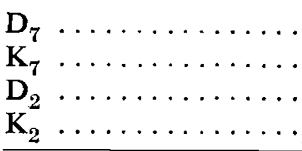 & $\begin{array}{l}0,32 \\
0,37 \\
0,39 \\
0,39\end{array}$ & $\begin{array}{l}0,32 \\
0,37 \\
0,35 \\
0,37\end{array}$ & $\begin{array}{l}0,015 \\
0,012 \\
0,031 \\
0,027\end{array}$ & $\begin{array}{l}0,027 \\
0,018 \\
0,054 \\
0,051\end{array}$ & $\begin{array}{l}0,035 \\
0,029 \\
0,065 \\
0,062\end{array}$ \\
\hline
\end{tabular}

Dans la population Kaduna, les héritabilités réalisées $(0,37)$ ne diffèrent pas significativement de sa valeur estimée dans la population $(0,39)$, ainsi que dans d'autres expériences où la même population a été utilisée (LóPEZ-FANJuL \& HiLl, 1973). Ce n'est pas le cas pour la population Dahomey, où les héritabilités réalisées $(0,32$ et 0,35 ) sont significativement différentes et très inférieures à sa valeur estimée dans la population $(0,61)$.

Les intensités de sélection observées, calculées par la différentielle de sélection en écart-type à chaque génération, ainsi que ses valeurs espérées, calculées comme la moyenne de nombres ordonnés, sont présentées dans le tableau 5. Les valeurs observées restent constantes dans le temps et sont de l'ordre de 90 p. 100 et de 84 p. 100 des valeurs espérées dans les lignées sélectionnées avec des taux de $7 / 20$ et $2 / 20$, respectivement. 


\section{TABLEAU 5}

Intensité de sélection par génération et groupe de lignées

(valeurs espérées : $D_{7}=K_{7}=1,04 ; D_{2}=K_{2}=1,69$ ).

Selection intensities per generation and group of lines (expected values : $D_{7}=K_{7}=1.04 ; D_{2}=K_{2}=1.69$ ).

\begin{tabular}{|c|c|c|c|c|}
\hline \multirow{2}{*}{$\begin{array}{l}\text { Génération } \\
\text { Generation }\end{array}$} & \multicolumn{4}{|c|}{$\begin{array}{l}\text { Groupe de lignées } \\
\text { Group of lines }\end{array}$} \\
\hline & $\mathrm{D}_{7}$ & $\mathrm{~K}_{7}$ & $\mathrm{D}_{2}$ & $\mathbf{K}_{2}$ \\
\hline 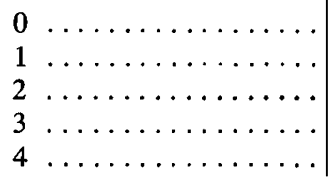 & $\begin{array}{l}0,96 \\
0,94 \\
0,91 \\
0,95 \\
0,96\end{array}$ & $\begin{array}{l}0,96 \\
0,95 \\
0,95 \\
0,97 \\
0,95\end{array}$ & $\begin{array}{l}1,39 \\
1,40 \\
1,44 \\
1,32 \\
-\end{array}$ & $\begin{array}{l}1,46 \\
1,43 \\
1,41 \\
1,49 \\
-\end{array}$ \\
\hline
\end{tabular}
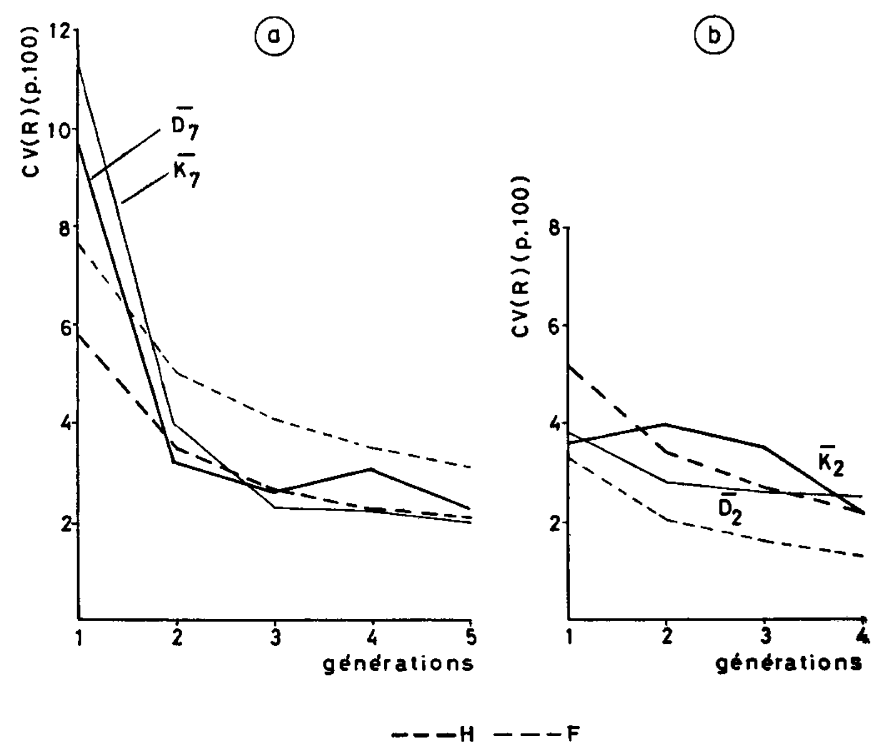

(b)

FIG. 2

Évolution du coefficient de variation $[\mathrm{CV}(\mathrm{R})](p .100)$ de la réponse à la sélection observée et sa valeur espérée avec sélection (F) et sans sélection $(\mathrm{H})$.

Evolution of the observed coefficient of variation of selection response [CV(R)] (p. 100) and its expected value under selection $(F)$ and without selection $(\mathrm{H})$.

a) Groupes de lignées $D_{7}$ et $K_{7}$. Groups of lines $D_{7}$ and $K_{7}$. b) Groupes de lignées $D_{2}$ et $K_{2}$. Groups of lines $D_{\varepsilon}$ and $K_{2}$. 


\section{4. - Variabilité de la réponse à la sélection}

Dans la figure 2 on présente les valeurs observées du coefficient de variation de la réponse pour chacun des quatre types de lignées et ses valeurs espérées avec sélection (F) (HILL, 1974) ou sans sélection (H) (WRIGHT, 1952). Dans les deux prédictions on a utilisé pour les deux populations la valeur de l'héritabilité estimée dans la population Kaduna $(0,39)$ et des effectifs génétiques constants et égaux au nombre d'individus sélectionnés dans chaque cas. Pour un même nombre d'individus sélectionnés le comportement des deux populations est très semblable, et proche de celui prédit sans sélection. La prédiction avec sélection s'écarte considérablement de l'observation; dans le cas des lignées sélectionnées avec le taux 7/20 elle est surestimée, et pour le taux $2 / 20$ elle est sous-estimée.

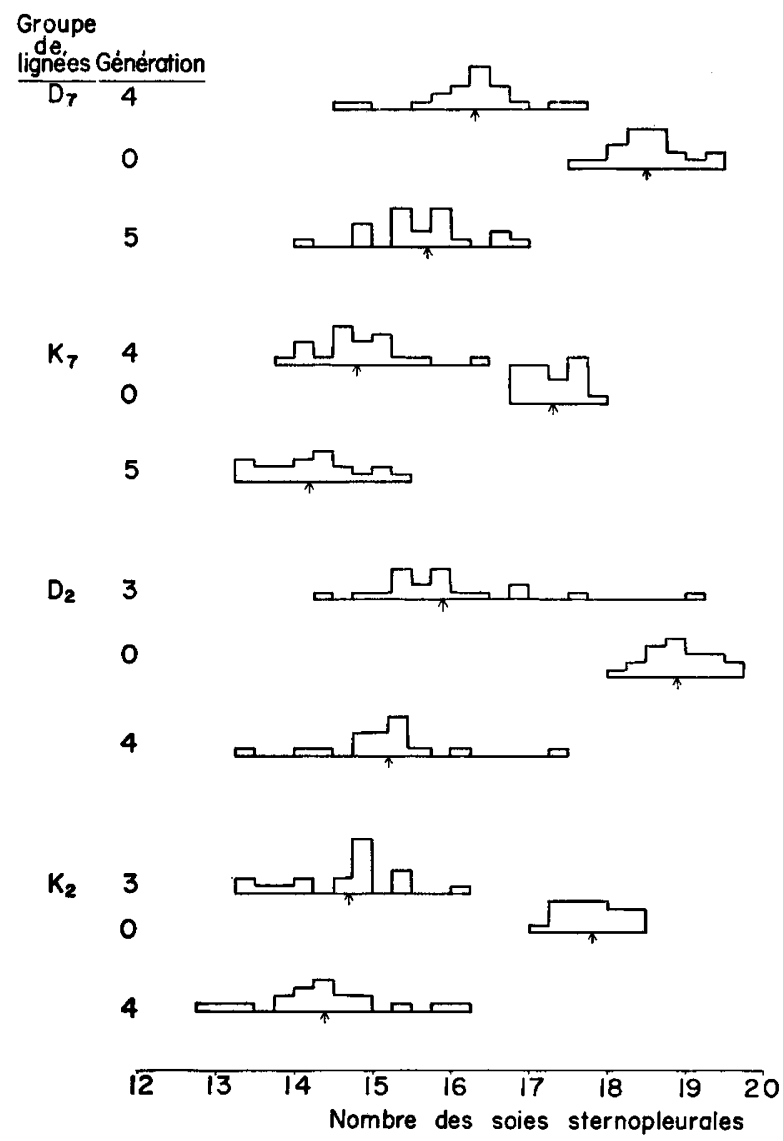

FIG. 3

Distribution des moyennes des lignées sélectionnées dans la génération 0 et les deux dernières générations (3-4 ou 4-5) par groupe de lignées.

Distribution of the means of the selected lines in generation 0 and in the last two generations (3-4 or 4-5) for each group of lines. 
Afin de donner une meilleure représentation des résultats obtenus, on présente dans la figure 3 la distribution des moyennes des lignées sélectionnées dans la génération 0 et les deux dernières générations de sélection (3-4 ou 4-5) pour chaque groupe de lignées. On peut constater que la variation de cette distribution dans les générations finales est toujours supérieure à celle de la génération initiale. Par ailleurs, cette variation aussi est supérieure dans les groupes de lignées où le nombre d'individus sélectionnés est plus bas $\left(D_{2}, K_{2}\right)$ par rapport aux autres groupes $\left(D_{7}, K_{7}\right)$. Pour un même taux de sélection la variation des moyennes des lignées sélectionnées dans les deux populations est comparable.

\section{Discussion}

L'interprétation de nos résultats demande une analyse préalable de deux problèmes. Le premier est celui que pose la forte diminution observée de la variance phénotypique des lignées sélectionnées, ce qui est en contradiction avec une des hypothèses du modèle de prédiction utilisé. $\mathrm{Ce}$ phénomène est couramment observé dans les expériences de sélection et, en ce qui concerne le caractère étudié, on peut l'attribuer, tout au moins en partie, à un effet d'échelle et même à la particularité qu'ont les données discrètes par rapport aux continues, notamment la dépendance entre les moyennes et les variances. Cet effet d'échelle pourrait être corrigé par une transformation logarithmique qui, dans notre cas, nous donnerait une variance phénotypique finale de l'ordre de 70 à 80 p. 100 de cette initiale, tandis que si l'on considère les données avant transformation, la variance phénotypique finale n'est que de l'ordre de 30 p. 100 de celle initiale. Nous avons préféré, toutefois, analyser la variabilité de la réponse en fonction du coefficient de variation, puisque celui-ci est indépendant de la variance, si l'héritabilité reste constante tout au long du processus sélectif ; cette indication nous est donnée par la linéarité de la réponse observée. L'utilisation du modèle de Hill $(1974,1977)$ dans ce contexte semble donc correcte.

Le second problème est posé par la différence observée entre la valeur estimée de l'héritabilité de la population Dahomey $(0,61)$ et les héritabilités réalisées correspondantes $(0,32 ; 0,35)$. Une différence semblable a eu lieu également dans une autre expérience (GALlego \& López-FANJUl, en préparation) dans laquelle, l'héritabilité, dans un échantillon différent de la même population Dahomey, a été estimée à $0,59 \pm 0,05$ et l'héritabilité réalisée globale correspondante à un groupe de six lignées Dahomey sélectionnées individuellement avec le taux 6/60 pendant six générations, a été estimée à $0,36 \pm 0,03$. Dans les deux cas nous avons pu vérifier que la réponse n'a pas été significativement asymétrique. La répétabilité apparente du phénomène n'amoindrit pas le caractère énigmatique du fait. Malheureusement, nous n'avons pas été capables de l'expliquer de façon satisfaisante. Etant donné la similitude existante entre les réponses à la sélection observées dans les deux populations, nous sommes enclins à considérer que l'héritabilité des deux populations est semblable, et en conséquence nous avons utilisé dans les équations de prédiction la variabilité de la réponse, la valeur de l'héritabilité estimée dans la population Kaduna $(0,39)$; ceci réduit notre propos initial qui prétendait étudier le phénomène dans deux populations ayant une héritabilité différente. La prédiction de la réponse à la sélection dans la population Kaduna est en accord avec les résultats obtenus, ce qui est impliqué par la concordance existante entre la valeur de l'héritabilité estimée dans 
la population et celles réalisées dans les lignées sélectionnées, ainsi que par celle qu'on trouve entre les valeurs de l'intensité de sélection observées et espérées.

HILl (1972 a, b) a démontré que l'écart-type du coefficient de régression linéaire de la réponse cumulée sur la différentielle de sélection cumulée, est une sous-estimation de l'erreur d'estimation réelle, car la variance der valeurs moyennes des lignées sélectionnées augmente à cause de la dérive génétique et aussi, parce que les moyennes dans les différentes générations sont corrélées. Le modèle de Hill suppose la constance des variances génétique et phénotypique, et doit être appliqué uniquement aux expériences de sélection à court terme. Nos données montrent de façon constante dans chacun des quatre groupes de lignées considérées, que l'estimation empirique de l'écart-type de l'héritabilité réalisée $\left(s_{b}^{-}\right)$est toujours inférieure, non seulement à la valeur prédite selon le modèle de Hill $\left(s_{b c}^{\prime}\right)$ mais aussi à l'écart-type du coefficient de régression mentionné $\left(s_{b c}\right)$. Ceci peut être attribué à la forte diminution de la variance phénotypique des lignées sélectionnées ainsi qu'au fait que la variance inter lignées prédite sous l'hypothèse de sélection est inférieure à sa valeur observée. Les deux causes supposent l'obtention d'une estimation empirique de l'écart-type de l'héritabilité réalisée qui est inférieure à sa valeur espérée. De toute façon, nos résultats mettent en évidence que la correction de Hill ne doit pas être utilisée sans discernement.

HiLl (1977) fait remarquer au moment de prédire la variabilité de la réponse à la sélection, qu'il convient plus d'utiliser dans la pratique les formules correspondantes à la variation espérée du fait de la seule action de la dérive génétique, à cause de l'autocorrélation existante entre les variances génétiques d'une lignée dans différentes générations de sélection. Bien que nous n'ayons pas pu mettre en évidence cette corrélation à cause du petit nombre de degrés de liberté existant, nos observations vont dans le sens de la remarque de Hill. Il existe toujours un accord admissible entre les observations et les prédictions faites suivant un modèle dans lequel la dérive génétique est le seul agent modificateur des fréquences génétiques des loci additifs et indépendants.

Nous avons supposé que l'effectif génétique d'une lignée est égal au nombre d'individus sélectionnés, et bien que dans la pratique cet effectif soit normalement inférieur, cela n'a pas une grande importance quant à la prédiction de la variabilité de la réponse à la sélection, étant donnée la valeur de l'héritabilité du caractère étudié. $\mathrm{Au}$ contraire, la prédiction des valeurs du coefficient de variation de la variance phénotypique dépend substantiellement de la valeur réelle de l'effectif génétique et, si le nombre d'individus sélectionnés est très petit, comme dans les groupes de lignées $\mathrm{D}_{2}$ et $\mathrm{K}_{2}$, la qualité de la prédiction est considérablement détériorée, car les hypothèses du modèle utilisé (l'indépendance tout spécialement) seront difficilement réalisables.

Reçu pour publication le 31 mars 1982.

\section{Remerciements}

Nous tenons à remercier R. Diez Barra, du Département de Génétique de l'I.N.I.A. à Madrid, pour la réalisation des programmes sur ordinateur. 


\section{Summary \\ An experimental study of the variability of selection response in Drosophila melanogaster}

Short-term response to selection for low sternopleural bristle number in D. melanogaster was analysed in two populations (Kaduna and Dahomey) and for two proportions selected $(10$ p. 100 and 35 p. 100). In each of the four possible cases (populations $\times$ proportions selected) twenty lines were selected during a period of 4-5 generations.

Realised heritabilities did not significantly differ from its estimated value in the Kaduna base population, but they were considerably smaller than those estimated in the Dahomey base population. The empirical standard errors of those realised heritabilities were substantially smaller than their predicted value corrected for drift. The suggestion is made that an a priori correction should not be made.

A satisfactory agreement was found between the observed value of the coefficient of variation of selection response and that predicted under a model in which the genetic variance of the trait is considered to be due to the segregation of a number of additive and independent loci, and the environmental variance is assumed to be the same in all generations, in populations where genetic drift is the only force acting on gene frequency. The values of the coefficient of variation of the phenotypic variance were also predicted under the same model and a good fit was found for those lines where the selected proportion was 35 p. 100. The quality of this prediction deteriorated for lines selected at 10 p. 100, probably due to their very small effective population size. Our results suggest that in short-term selection experiments the value of those coefficients of variation in selected lines can be reasonably approximated by the prediction made for unselected lines.

\section{Références bibliographiques}

Bulmer M.G., 1971. The effect of selection on genetic variability. Am. Nat., 105, 201-211. Clayton G.A., Morris J.A., Robertson A., 1957. An experimental check on quantitative genetical theory. 1. Short-term responses to selection. J. Genet., 55, 131-151.

FALCONER D.S., 1973. Replicated selection for body weight in mice. Genet. Res. Camb., 22, 291-321.

Hill W.G., 1970. Design of experiments to estimate heritability by regression of offspring on selected parents. Biometrics, 26, 565-571.

HiLl W.G., 1972 a. Estimation of realised heritabilities from selection experiments. I - Divergent selection. Biometrics, 28, 741-765.

HILL W.G., 1972 b. Estimation of realised heritabilities from selection experiments. II - Selection in one direction. Biometrics, 28, 767-780.

HILL W.G., 1974. Variability of response to selection in genetic experiments. Biometrics, 30, 363-366.

Hill W.G., 1977. Variation in response to selection. In : Pollak, Kempthorne et Bailey, Proceedings of the International Conference on Quantitative Genetics, 343-365, The Iowa State University Press, Ames, Iowa.

López-Fanjul C., Hill W.G., 1973. Genetic differences between populations of Drosophila melanogaster for a quantitative trait. I - Laboratory populations. Genet. Res. Camb., 22, 51-68.

RoBERTSON A., 1967. The nature of quantitative genetic variation. In : BRINK, Heritage from Mendel, 265-280, The University of Wisconsin Press, Madison, Wisconsin.

Robertson A., 1968. The spectrum of genetic variation. In : LewonTin, Population Biology and Evolution, 5-16, Syracuse University Press, Syracuse, New York.

Robertson A., 1970. Some optimum problems in individual selection. Theor. Pop. Biol., $1,120-127$.

Toro M.A., 1978. Diferencias genéticas entre problaciones de Tribolium castaneum para caracteres cuantitativos. Tesis doctoral, Universidad Complutense de Madrid.

Wright S., 1952. The theoretical variance within and among subdivisions of a population that is in steady state. Genetics, 37, 312-321.

YUKSEL E., 1974. Check on a quantitative genetic theory. M.Sc. thesis, University of Edinburgh. 